\title{
GCPW-to-SIW Transition Using Tapered Slot
}

\author{
Chia-Fen Liu, Yi-Hsuan Lu, and Chun-Long Wang \\ Electronics Engineering, National Taiwan University of Science and Technology \\ 106 Keelung Rd., Sec. 4, No. 43, Taipei, Taiwan \\ M9502205@mail.ntust.edu.tw; yvonne850306@gmail.com; clw@mail.ntust.edu.tw
}

\begin{abstract}
In this paper, the GCPW-to-SIW transition using the tapered slot is utilized to enhance the bandwidth of the GCPW-to-SIW transition using the uniform slot. By tapering the slot width of the uniform slot, the bandwidth of the transition would be increased. The fractional bandwidth of the GCPW-to-SIW transition using the tapered slot is $42 \%$ and the corresponding insertion loss in this frequency range is smaller than $0.2 \mathrm{~dB}$.
\end{abstract}

Keywords: GCPW, SIW, Transition, Tapered slot.

\section{Introduction}

Planar transmission lines, which include the microstrip line, coplanar waveguide (CPW), coplanar stripline, and slotline, etc., have been commonly used in the realization of various microwave components due to their characteristics of compact size and cheap fabrication cost. Among these planar transmission lines, coplanar waveguide [1] is widely applied in the implementation of various microwave circuits, including antennas [2]-[3] and filters [4]-[5] since it is advantageous of low dispersion and easy integration as compared with other types of planar transmission lines.

Rectangular waveguide has been commonly used to realize various microwave components with high Q-factors since it has the characteristics of low loss and high power capability. However, as compared with the planar transmission lines, the rectangular waveguide is bulky in size and expensive in cost.

In order to retain both the advantages of the planar transmission line and rectangular waveguide, a new planar structure named the substrate integrated waveguide (SIW) is proposed [6]-[8]. Since the substrate integrated waveguide is the planar form of the rectangular waveguide, which could be realized through the conventional printed circuit board (PCB) process, it would possess the attributes of planar transmission lines, which are low cost and easy implementation. Besides, since the substrate integrated waveguide is in the form of the rectangular waveguide, its Q-factor is much higher than that of the planar transmission line. As a result, it could be used to realize various microwave components with middle Q-factors.

In order to integrate the microwave components realized in the forms of planar transmission lines and substrate integrated waveguide, a variety of CPW-to-SIW transitions are proposed [9]-[12]. At first, a CPW-to-SIW transition using the quarter-wavelength stub is proposed [9]. Since the electric field distribution of the quarter-wavelength stub is matched to that of the $\mathrm{TE}_{10}$ mode of the SIW, easy integration of the CPW and SIW could be realized. Although the transition can be easily implemented, the bandwidth of the transition is narrow. In order to accomplish a broadband CPW-to-SIW transition, some researchers use the probe to excite the magnetic field of the $\mathrm{TE}_{10}$ mode of the SIW [10]. Although the transition has a broadband response and a compact size, the performance of the transition is sensitive to the size of the probe, which means that the dimensions of the probe must be precisely controlled. Besides, other researchers use the slot dipole along with the resonator to transfer the energy from the CPW to the SIW [11]. Although the transition has a broadband response, it has a large size. In order to reduce the size of the transition, a compact and broadband GCPW-to-SIW transition using the quarterwavelength transformer with the $90^{\circ}$-bent slot is proposed [12]. Even though the size of the transition is compact, the bandwidth of the transition is unable to cover the whole frequency range of the rectangular waveguide band. In order to enhance the bandwidth of the transition without further increasing the size of the transition, a GCPW-to-SIW transition using the tapered slot is proposed in this paper. 


\section{GCPW-to-SIW Transition Using Uniform Slot}

The schematic view of the GCPW-to-SIW transition using the uniform slot is shown in Fig. 1 where the substrate used is FR4 with a thickness of $0.508 \mathrm{~mm}$ and a relative dielectric constant of 2.2. The SIW is formed by placing vias between the top and bottom metals of the substrate. The diameter of the via is chosen to be $0.3 \mathrm{~mm}$ so as to accommodate the via diameter to the PCB fabrication limit provided by the vendor. The distance between adjacent vias is chosen to be $0.3 \mathrm{~mm}$ so that the energy may not leak out of the SIW from the slits between the vias. The remaining dimensions of the transition are shown in Table I.

In order to investigate the performance of the transition, Fig. 1 along with Table 1 is simulated by the Ansoft HFSS and the frequency response is shown in Fig. 2. As can be seen from Fig. 2, the fractional bandwidth for which the return loss is larger than $15 \mathrm{~dB}$ is estimated to be $38 \%$ and the corresponding insertion loss in this frequency range of the fractional bandwidth is smaller than $0.2 \mathrm{~dB}$.

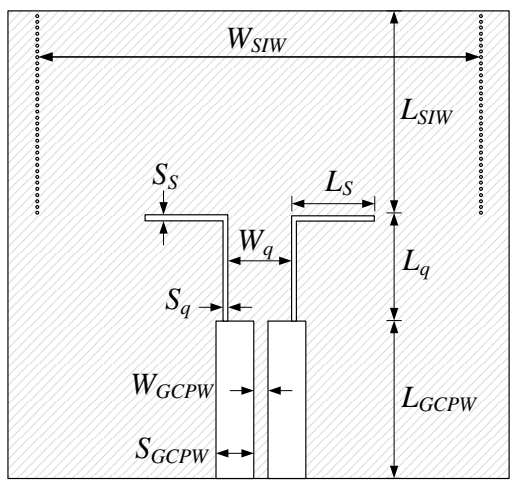

Fig. 1: The schematic view of the GCPW-to-SIW transition using the uniform slot.

Table 1: Dimensions for the GCPW-to-SIW transition using the uniform slot.

\begin{tabular}{|c|c|c|c|c|c|}
\hline$W_{G C P W}$ & $S_{G C P W}$ & $L_{G C P W}$ & $W_{q}$ & $S_{q}$ & $L_{q}$ \\
\hline 1.5 & 4.0 & 20.0 & 7.2 & 0.2 & 12.0 \\
\hline$S_{S}$ & $L_{S}$ & $W_{S I W}$ & $L_{S I W}$ & & \\
\hline 0.2 & 8.5 & 48.9 & 26.7 & & \\
\hline
\end{tabular}

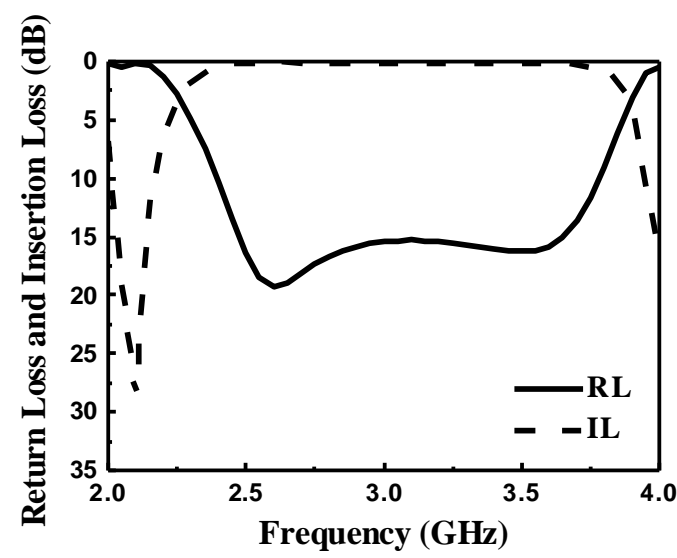

Fig. 2: Frequency responses of the return and insertion losses for the GCPW-to-SIW transition using the uniform slot.

\section{GCPW-to-SIW Transition Using Tapered Slot}

In order to enhance the bandwidth of the GCPW-to-SIW transition using the uniform slot, the GCPW-to-SIW transition using the tapered slot is proposed as shown in Fig. 3. 


\subsection{Parameter Study of the Transition}

In order to investigate the performance of the GCPW-to-SIW transition using the tapered slot, Fig. 3 along with Table 1 is simulated by the Ansoft HFSS through varying the stub angle $\theta$. The frequency responses of the return and insertion losses with various stub angles $\theta$ are shown in Fig. 4. As can be seen from Fig. 4, the transition with a stub angle $\theta=21.0^{\circ}$ has the optimal response where the fractional bandwidth of the transition is $42 \%$ and the corresponding insertion loss is smaller than $0.2 \mathrm{~dB}$.

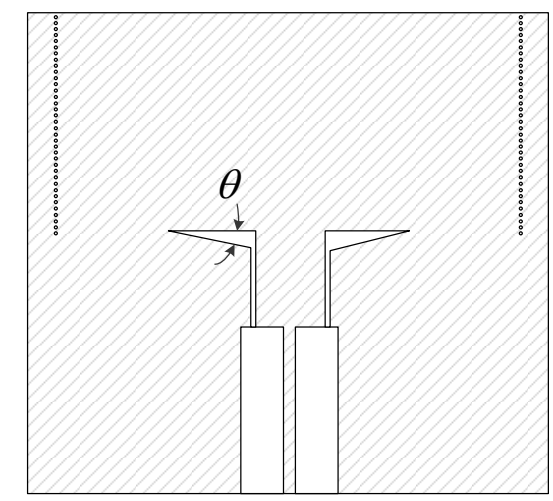

Fig. 3: The schematic view of the GCPW-to-SIW transition using the tapered slot.

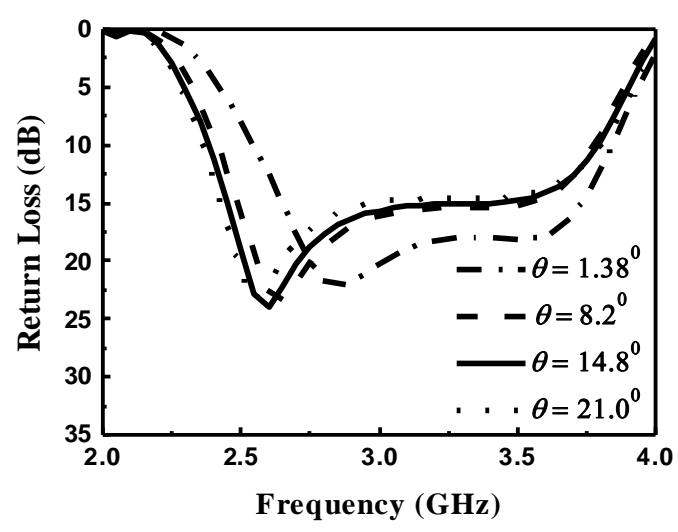

(a)

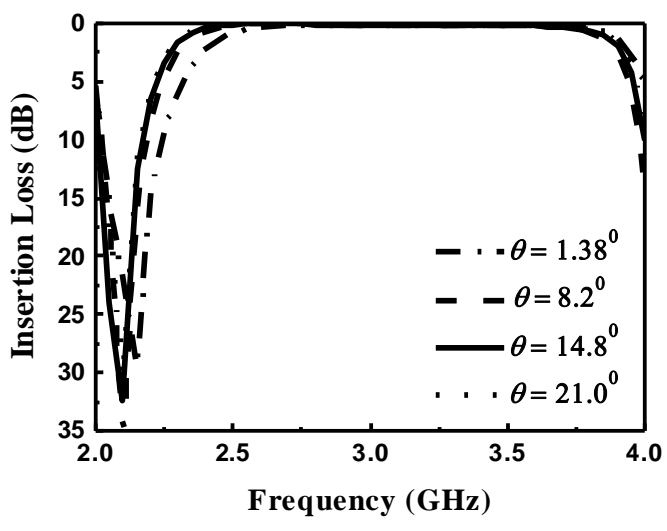

(b)

Fig. 4: Frequency responses of the return and insertion losses for the GCPW-to-SIW transition using the tapered slot with various stub angles $\theta$. (a) Frequency response of the return loss. (b) Frequency response of the insertion loss.

\subsection{Back-to-Back Verification}

In order to verify the simulation result of the GCPW-to-SIW transition using the tapered slot, two GCPW-to-SIW transitions using the tapered slot are connected in a back-to-back form as shown in Fig. 5. The length of the SIW between two transitions is $L_{b a c k}=53.3 \mathrm{~mm}$. Fig. 5 is then simulated by the Ansoft HFSS and the frequency responses of the return and insertion losses are shown in Fig. 6. In order to verify the simulation results of Fig. 6, the back-to-back transition shown in Fig. 5 is fabricated as shown in Fig. 7. Fig. 7 is then measured by Anritsu 37347C VNA after this equipment is calibrated with the TRL calibration kit. The measured frequency responses of the return and insertion losses are also shown in Fig. 6. The reasonable agreement between the simulation and measurement results shown in Fig. 6 verifies the correctness of the transition design. 


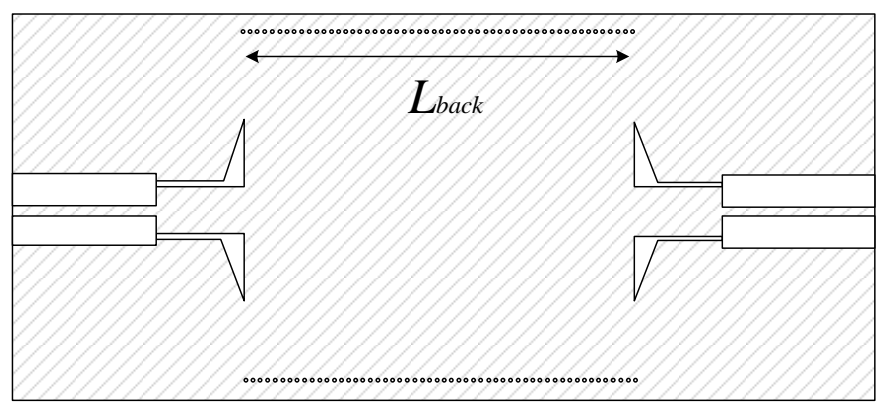

Fig. 5: The schematic view of the back-to-back GCPW-to-SIW transition using the tapered slot.

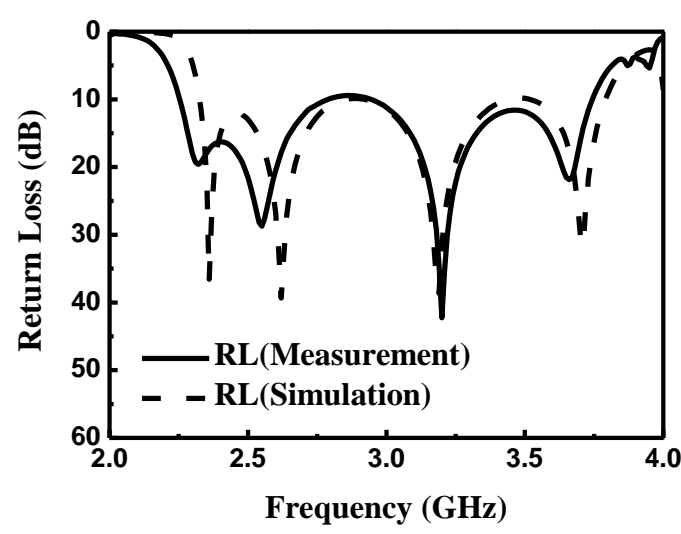

(a)

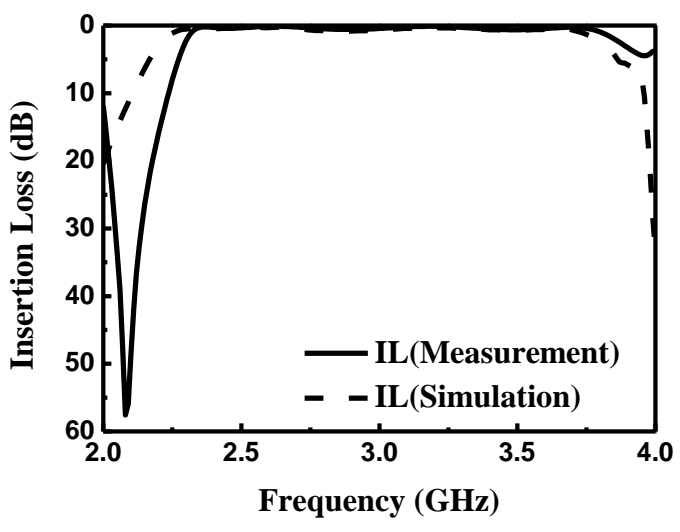

(b)

Fig. 6: Comparison between the simulation and measurement results of the back-to-back GCPW-to-SIW transition using the tapered slot. (a) Frequency response of the return loss. (b) Frequency response of the insertion loss.

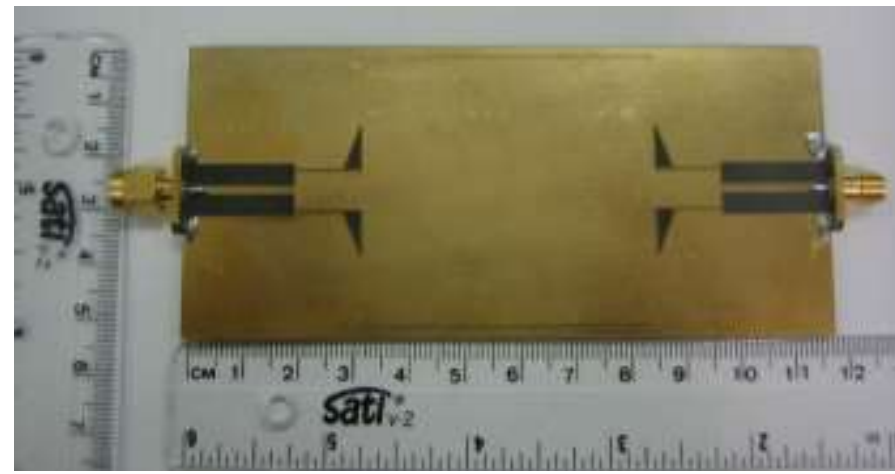

Fig. 7: The fabricated circuit of the back-to-back GCPW-to-SIW transition using the tapered slot.

\section{Conclusion}

In this paper, a GCPW-to-SIW transition using the tapered slot is proposed to enhance the fractional bandwidth of the GCPW-to-SIW transition using the uniform slot. As for the GCPW-to-SIW transition using the uniform slot, the fractional bandwidth for which the return loss is larger than $15 \mathrm{~dB}$ is estimated to be $38 \%$ and the corresponding insertion loss in this frequency range of the fractional bandwidth is smaller than $0.2 \mathrm{~dB}$. Through using the tapered slot, the fractional bandwidth could be increased to be $42 \%$ while the corresponding insertion loss in this frequency range and the circuit size is unaltered. 


\section{Acknowledgements}

This work was supported in part by the Ministry of Science and Technology, Taiwan, under Grant MOST-107-2221-E011-052.

\section{References}

[1] R. N. Simons, Coplanar Waveguide Circuits, Components, and Systems. John Wiley \& Sons, Inc., pp. 108, 2001.

[2] R. Fu and L. Zhu, "Printed U-shaped slot antennas fed by CPW via inductive coupling," IEEE International Workshop. Antenna Tech., pp. 303-306, Mar. 2005.

[3] J.-H. Lu, "Novel compact design of CPW-fed circular patch antenna," IEEE Trans. Antenna Propag., vol. 1, pp. 34-37, Jun. 2002.

[4] X.-D. Huang and C.-H. Cheng, "A novel coplanar-waveguide bandpass filter using a dual-mode square-ring resonator," IEEE Microw. Wireless Compon. Lett., vol. 16, pp. 13-15, Jan. 2006.

[5] Y.M. Bo, C.-H. Cheng, and X.-D. Huang, "A novel bandpass filter using a dual-mode square patch resonator with combined CPW and microstrip input/output," IEEE Asia-Pacific Conf., pp. 802-805, Aug. 2006.

[6] F. Xu and K. Wu, "Guided-wave and leakage characteristics of substrate integrated waveguide," IEEE Trans. Microw. Theory Tech., vol. 53, pp. 66-73, Jan. 2005.

[7] H. Nam, T.-S. Yun,K.-B. Kim, K.-C. Yoon, and J.-C. Lee, "Ku-band transition between microstrip and substrate integrated waveguide (SIW)," IEEE Asia-Pacific Microw. Conf., vol. 1, Dec. 2005.

[8] T. Kai, J. Hirokawa, and M. Ando, "A stepped post-wall waveguide with aperture interface to standard waveguide," IEEE 2004 Antenna and Propag. Society Int. Symposium, vol. 2, pp. 1527-1530, Jun. 2004.

[9] D. Deslandes and K. Wu, "Integrated transition of coplanar to rectangular waveguides," IEEE MTT-S Int., vol. 2, pp. 619-622, May 2001.

[10] D. Deslandes and $\mathrm{K}$. Wu, "Analysis and design of current probe transition from grounded coplanar to substrate integrated rectangular waveguides," IEEE Trans. Microw. Theory Tech., vol. 53, pp. 2487-2494, Aug. 2005.

[11] A. Patrovsky, M. Daigle, and K. Wu, "Millimeter-wave wideband transition from CPW to substrate integrated waveguide on electrically thick high-permittivity substrates," IEEE Microw. Conf., pp. 138-141, Oct. 2007.

[12] R.-Y. Fang, C.-F. Liu, and C.-L. Wang, "Compact and broadband CB-CPW-to-SIW transition using stepped-impedance resonator with $90^{\circ}$-bent slot," IEEE Trans. Components, Packaging, and Manufacturing Technology, vol. 3, no. 2, pp. 247-252, Feb. 2013. 